21st Particles and Nuclei International Conference (PANIC 2017)

International Journal of Modern Physics: Conference Series

Vol. 46 (2018) 1860024 (6 pages)

(C) The Author(s)

DOI: $10.1142 / \mathrm{S} 2010194518600248$

\title{
XYZ States at BESIII
}

\author{
Junhao Yin (on behalf of the BESIII collaboration) \\ Institute of High Energy Physics, CAS \\ $19 B$ Yuquan Road, Beijing City, 100049, China \\ yinjh@ihep.ac.cn
}

Published 3 May 2018

\begin{abstract}
The BESIII experiment have collected about $9 \mathrm{fb}^{-1}$ luminosity data samples above $3.8 \mathrm{GeV}$. Recent result on the XYZ states, which are also called exotic states, from BESIII experiment is presented in this talk, including the measurement of resonance parameters of vector $Y$ states, the establishment of two isospin triplets of $Z_{c}$ states, and the determination of spin-parity of $Z_{c}^{ \pm}(3900)$.
\end{abstract}

Keywords: Exotic states; Charmonium; Charmonium-like.

\section{Introduction}

The potential model works well in describing the heavy quarkonia states, especially for the charmonia states below the open-charm threshold. However, above this threshold, there are still many predicted states have not been observed yet. In recent years, an abundance of states have been discovered above the open charm threshold, which take advantage of the large statistics from B factory and BESIII experiment. Most of them are beyond the theoretical predictions and with more complex substructures. All of these states are called exotic states. Those states are mostly found in reconstructing the $c \bar{c}$ final states, so they are also called as charmonium-like states or $X Y Z$ states.

\section{Results on $Y$ states}

$Y(4260)$ is the first $Y$ state with $J^{P C}=1^{--}$observed by $B A B A R$ experiment in the ISR process $e^{+} e^{-} \rightarrow \gamma_{I S R} \pi^{+} \pi^{-} J / \psi^{1}$. This observation was subsequently confirmed by CLEO ${ }^{2}$ and Belle ${ }^{3}$ experiments. Soon after, the evidence of $Y(4360)$ state is found in $\pi^{+} \pi^{-} \psi^{\prime}$ spectrum by $B A B A R^{4}$ and confirmed by Belle ${ }^{5}$. Recently, BESIII

This is an Open Access article published by World Scientific Publishing Company. It is distributed under the terms of the Creative Commons Attribution 4.0 (CC-BY) License. Further distribution of this work is permitted, provided the original work is properly cited. 
experiment confirms $Y$ (4360) lineshape in $e^{+} e^{-} \rightarrow \pi^{+} \pi^{-} \psi^{\prime 6}$ and $e^{+} e^{-} \rightarrow \pi^{0} \pi^{0} \psi^{\prime}$ modes ${ }^{7}$, as shown in Fig. 1, with much better precision. A fit to the cross section from the charged mode indicates that there might be two structure contributions. The mass and width of the first resonance are measured to be $(4209.5 \pm 7.4 \pm$ 1.4) $\mathrm{MeV} / \mathrm{c}^{2}$ and $(80.1 \pm 24.6 \pm 2.9) \mathrm{MeV}$, and those of the second resonance are $(4383.8 \pm 4.2 \pm 0.8) \mathrm{MeV} / \mathrm{c}^{2}$ and $(84.2 \pm 12.5 \pm 2.1) \mathrm{MeV}$. The significance of $Y(4220)$ is determined to be $5.8 \sigma$. The evidence of the first resonance is reported for the first time and the measurement of the second resonance are consistent with Belle and BABAR results, but with much improved precision.
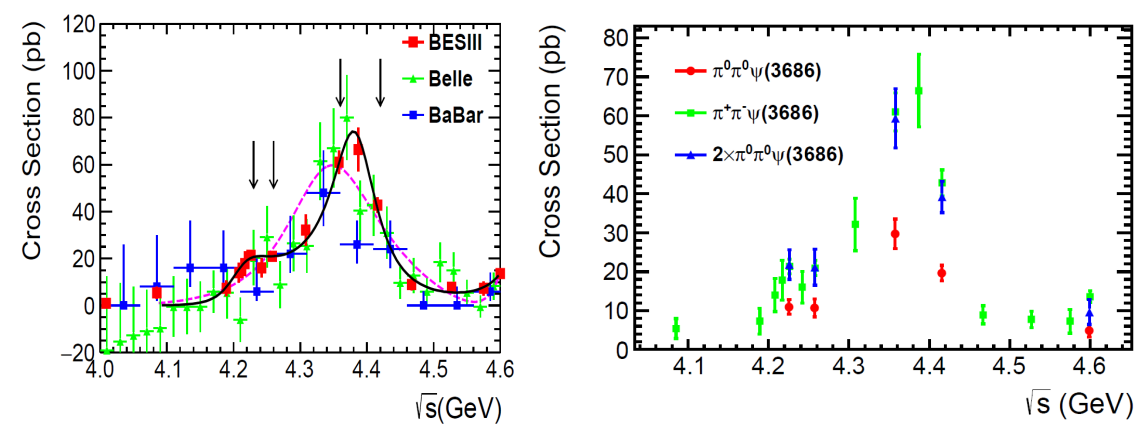

Fig. 1. Energy dependent cross section $\sigma^{B}\left[e^{+} e^{-} \rightarrow \pi^{+} \pi^{-} \psi^{\prime}\right]$ (left), red dots are the results obtained in BESIII, solid curve is the fit to BESIII results with the coherent sum of three BreitWigner functions, dashed curve is the fit with two Breit-Wigner without $Y(4220)$. Right plot shows the cross section $\sigma^{B}\left[e^{+} e^{-} \rightarrow \pi^{0} \pi^{0} \psi^{\prime}\right]$ and the comparison with $\sigma^{B}\left[e^{+} e^{-} \rightarrow \pi^{+} \pi^{-} \psi^{\prime}\right]$.

Recently, BESIII reported the most precise cross section measurements for $e^{+} e^{-} \rightarrow \pi^{+} \pi^{-} J / \psi$ from 3.77 to $4.6 \mathrm{GeV}^{8}$. The analysis is performed with 19 energy points with integrated luminosity of larger than $50 \mathrm{pb}^{-1}$ per point, which is called as $X Y Z$ data, and 103 energy points with integrated luminosity of about 8 $\mathrm{pb}^{-1}$ per point, which is called as R-scan data. The energy dependent Born cross sections of $e^{+} e^{-} \rightarrow \pi^{+} \pi^{-} J / \psi$ from the two kinds of data samples are shown in Fig. 2. A binned maximum likelihood fit with a coherent sum of three Breit-Wigner functions and an coherent $\psi(3770)$ component is performed simultaneously to the cross sections. Two resonant structures are observed. The mass and width of the first resonant structure are $(4220.0 \pm 3.1 \pm 1.4) \mathrm{MeV} / \mathrm{c}^{2}$ and $(44.1 \pm 4.3 \pm 2.0) \mathrm{MeV}$, respectively, which agree with those of $Y(4260)$ but much narrower width. The parameters of the second resonant structure are $(4320.0 \pm 10.4 \pm 7.0) \mathrm{MeV} / \mathrm{c}^{2}$ and $\left(101.4_{-19.7}^{+25.3} \pm 10.2\right) \mathrm{MeV}$. The statistical significance of the second resonance is $7.9 \sigma$. If we assume the two structures are same as $Y(4360)$, we observe a new decay channel of $Y(4360) \rightarrow \pi^{+} \pi^{-} J / \psi$ for the first time.

BESIII also report the analysis of process $e^{+} e^{-} \rightarrow \pi^{+} \pi^{-} h_{c}$ using $X Y Z$ data and R-scan data ${ }^{9}$. Energy dependent cross sections of $e^{+} e^{-} \rightarrow \pi^{+} \pi^{-} h_{c}$, which is shown in Fig. 3 can not be fitted with a single resonance. A binned maximum 

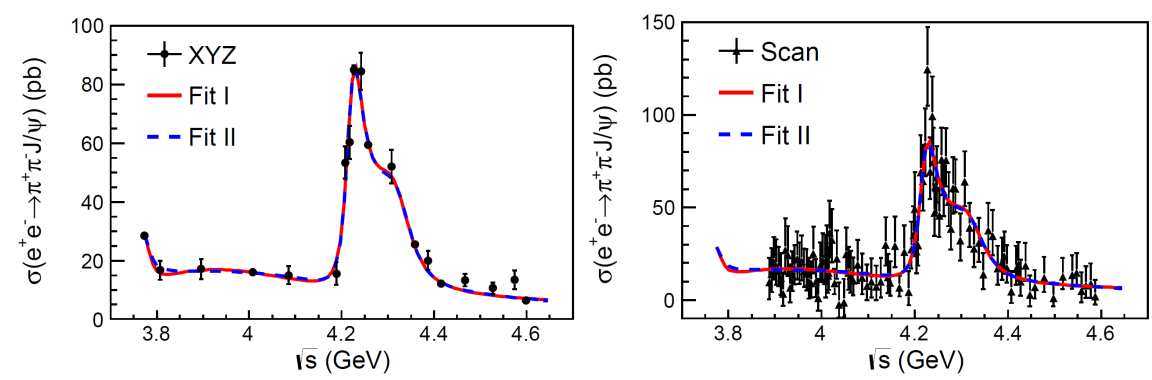

Fig. 2. Simultaneous fit to the energy dependent cross section $\sigma^{B}\left[e^{+} e^{-} \rightarrow \pi^{+} \pi^{-} J / \psi\right]$ measured from $X Y Z$ data (left) and R-scan data (right).

likelihood fit is performed to the cross sections with two structures. The statistical significance of the hypothesis of two structures over the hypothesis of only one structure is larger than $10 \sigma$. The mass and width of the first resonant structure are $\left(4218.4_{-4.5}^{+5.5} \pm 0.9\right) \mathrm{MeV} / \mathrm{c}^{2}$ and $\left(66.0_{-20.6}^{+16.2} \pm 0.4\right) \mathrm{MeV}$. The parameters of the second structure are $M=\left(4391.5_{-4.5}^{+6.3} \pm 0.9\right) \mathrm{MeV} / \mathrm{c}^{2}$ and $\Gamma=\left(139.5_{-20.6}^{+16.2} \pm 0.4\right) \mathrm{MeV}$. Likelihood contours in the mass and width planes of the measured two structures are provided in Fig. 3, together with the positions of $Y(4230), Y(4260), Y(4360)$, and $\psi(4415)$ with the parameters taken from the latest PDG average ${ }^{11}$. The low-lying resonance from the study of $\pi^{+} \pi^{-} J / \psi$ at BESIII ${ }^{8}$, marked as $Y(4260)^{\mathrm{BESIII}}$, is also compared. $Y(4260), Y(4360)$, and $\psi(4415)$ are located outside the $3 \sigma$ contours, while $Y(4230)$ and $Y(4260)^{\text {BESIII }}$ are overlapped with the $3 \sigma$ contour of $Y(4220)$.
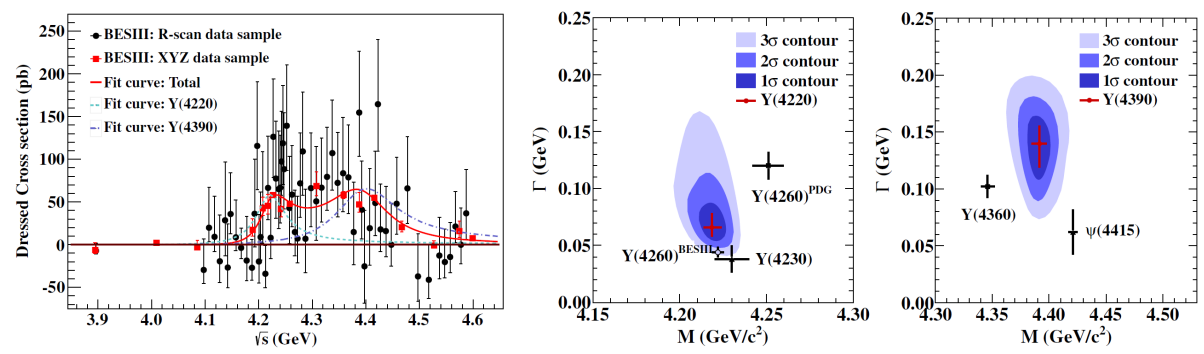

Fig. 3. (Fit to the dressed cross section of $e^{+} e^{-} \rightarrow \pi^{+} \pi^{-} h_{c}$ (left) with the coherent sum to two Breit-Wigner functions (solid curve). Dashed (dashed dot) curve shows the contribution from the two structure of $Y(4220)[Y(4390)]$. Middle and right plots show the likelihood contours in the mass and width planes for $Y$ (4220) (middle) and $Y$ (4390) (right).

BESIII also measured the cross sections of $e^{+} e^{-} \rightarrow \pi^{+} D^{0} D^{*-}$ from 4.05 to $4.60 \mathrm{GeV}$, as shown in Fig. 4, using $X Y Z$ data and R-scan data. A maximum likelihood fit is performed to the cross sections with two Breit-Winger and a coherent sum of three body phase space term. Two resonant structures are observed in the Born cross sections. The mass and width of the first resonance are measured to be $(4228.6 \pm 5.6 \pm 4.0) \mathrm{MeV} / \mathrm{c}^{2}$ and $(72.3 \pm 9.1 \pm 0.9) \mathrm{MeV}$, respectively, and those of 
the second resonance are $(4400.1 \pm 9.3 \pm 2.1) \mathrm{MeV} / \mathrm{c}^{2}$ and $(181.7 \pm 16.9 \pm 7.4) \mathrm{MeV}$. The statistical significance of the hypothesis with two structure over one structure is larger than $10 \sigma$. This result indicates that a new evidence for open-charm production associated with the $Y$ states is observed for the first time. The parameters of these two resonances are consistent with those measured in $e^{+} e^{-} \rightarrow \pi^{+} \pi^{-} h_{c}$, and the first resonance are also consistent with those measured in $e^{+} e^{-} \rightarrow \omega \chi_{c 0}{ }^{10}$ and $e^{+} e^{-} \rightarrow \pi^{+} \pi^{-} J / \psi$.

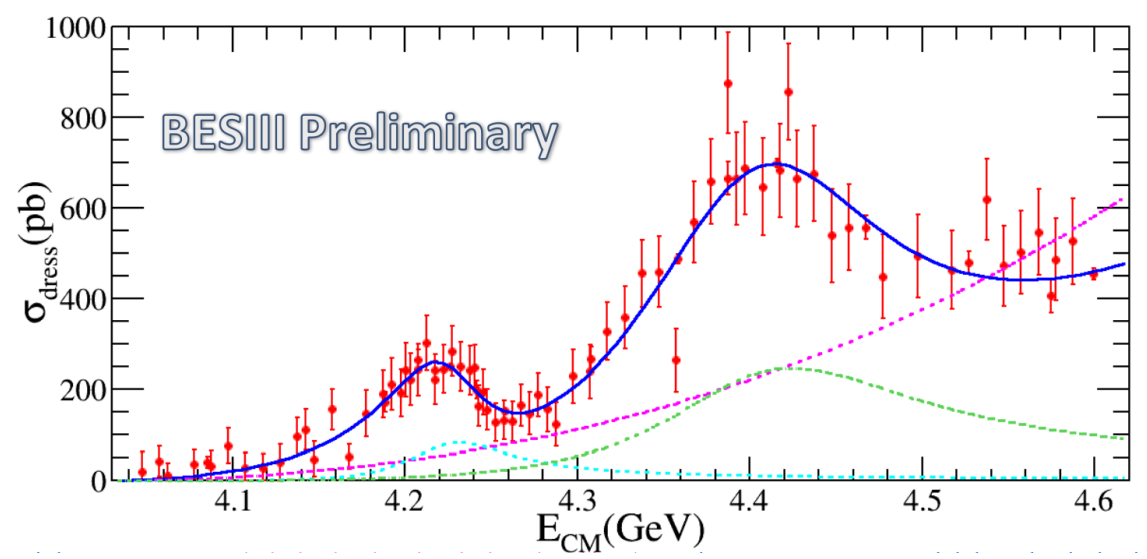

Fig. 4. Fit to the energy dependent dressed cross section $\sigma^{B}\left[e^{+} e^{-} \rightarrow \pi^{+} D^{0} D^{*-}\right]$ with a constant (pink dashed triple-dot) and two relativistic Breit-Wigner functions (green dashed double-dot and aqua dashed).

\section{Results on $Z$ states}

Till now, BESIII has observed quite a lot $Z$ states. Reconstructing the charmonium states such as $J / \psi$ or $h_{c}$, or the open charm final states, a lots of $Z_{c}$ states have been found $12,13,14,15,16,17,18,19$. According to the measured mass, we can classify these states into two isospin triplets, $Z_{c}(3900)$ in $\pi J / \psi$ and $D \bar{D}^{*}$ system, and $Z_{c}(4020)$ in $\pi h_{c}$ and $D^{*} \bar{D}^{*}$ system. In the analysis of $e^{+} e^{-} \rightarrow \pi^{+} \pi^{-} \psi^{\prime}$, BESIII also report the intermediate states searching ${ }^{6}$, and a narrow structure is observed at $4.416 \mathrm{GeV}$ as shown in Fig. 5. Similar structure is found in the neutral mode in $\pi^{0} \psi^{\prime}$ mass spectrum around $4.416 \mathrm{GeV}$. The measured parameters of the these states are listed in Table 1. It's interesting that if one can notice the relative branching fraction of $Z_{c}$ states decays into open charm meson pairs is much larger compared to branching fractions into charmonium states, which may indicate molecular contributions in the wave function. Question occurred that how to identify whether the states in one category are the same one.

To determine the spin parity of the $Z_{c}$ state, BESIII performed an amplitude analysis to the $e^{+} e^{-} \rightarrow \pi^{+} \pi^{-} J / \psi$ events with $1.92 \mathrm{fb}^{-1}$ data collected at $\sqrt{s}=4.23$ and $4.26 \mathrm{GeV}^{20}$. Four resonances, $\sigma, f_{0}(980), f_{2}(1270)$, and $f_{0}(1370)$, describing 


\begin{tabular}{c|c|c|c|c}
\hline \hline Name & Channel & mass $\left(\mathrm{MeV} / \mathrm{c}^{2}\right)$ & width $(\mathrm{MeV})$ & $\sigma^{\text {Born }}$ at $4.26 \mathrm{GeV}(\mathrm{pb})$ \\
\hline$Z_{c}(3900)$ & $\pi^{ \pm} J / \psi$ & $3899.0 \pm 3.6 \pm 4.9$ & $46 \pm 10 \pm 20$ & $13.5 \pm 5.2$ \\
& $\pi^{0} J / \psi$ & $3894.8 \pm 2.3 \pm 2.7$ & $29.6 \pm 8.2 \pm 8.2$ & $4.0 \pm 0.9$ \\
\hline$Z_{c}(3885)$ & $\left(D \bar{D}^{*}\right)^{ \pm}$ & $3881.7 \pm 1.6 \pm 1.6$ & $26.6 \pm 2.0 \pm 2.1$ & $108.4 \pm 6.9 \pm 8.8$ \\
& $\left(D \bar{D}^{*}\right)^{0}$ & $3885.7_{-5.7}^{+4.3} \pm 8.4$ & $35_{-12}^{+11} \pm 15$ & $47 \pm 9 \pm 10 \pm 0.9$ \\
\hline$Z_{c}(4020)$ & $\pi^{ \pm} h_{c}$ & $4022.9 \pm 0.8 \pm 2.7$ & $7.9 \pm 2.7 \pm 2.6$ & $7.4 \pm 1.7 \pm 2.1 \pm 1.2$ \\
& $\pi^{0} h_{c}$ & $4023.9 \pm 2.2 \pm 3.8$ & Fixed & $8.5 \pm 2.9 \pm 1.1 \pm 1.3$ \\
\hline$Z_{c}(4025)$ & $\left(D^{*} \bar{D}^{*}\right)^{ \pm}$ & $4026.3 \pm 2.6 \pm 3.7$ & $24.8 \pm 5.6 \pm 7.7$ & $89.0 \pm 18.7$ \\
& $\left(D^{*} \bar{D}^{*}\right)^{0}$ & $4025.5_{-4.7}^{+2.0} \pm 3.1$ & $23.0 \pm 6.0 \pm 1.0$ & $43.4 \pm 8.0 \pm 5.4$ \\
\hline$Z_{c}(4030)$ & $\pi^{ \pm} \psi^{\prime}$ & $4032.1 \pm 2.4$ & $26.1 \pm 5.3$ & - \\
& $\pi^{0} \psi^{\prime}$ & $4038.7 \pm 6.5$ & & - \\
\hline \hline
\end{tabular}

the $\pi^{+} \pi^{-}$mass spectrum, as well as $Z_{c}(3900)$ state and the non-resonant process are introduced in the fit. A simultaneous fit is performed to two data samples. When parameterizing the $Z_{c}^{ \pm}(3900)$ with a Flatte-like formula, the pole mass and width are measured to be $(3881.2 \pm 4.2 \pm 52.7) \mathrm{MeV} / \mathrm{c}^{2}$ and $(51.8 \pm 4.6 \pm 36.0) \mathrm{MeV}$, respectively. The quantum number of $Z_{c}(3900)$ is determined to be $1^{+}$with significance larger than $7.3 \sigma$ over other quantum number assumptions, which is a strong indication that $Z_{c}(3900)$ state found in $\pi J / \psi$ mode and $Z_{c}(3885)$ found in $D D^{*}$ mode are the same one.
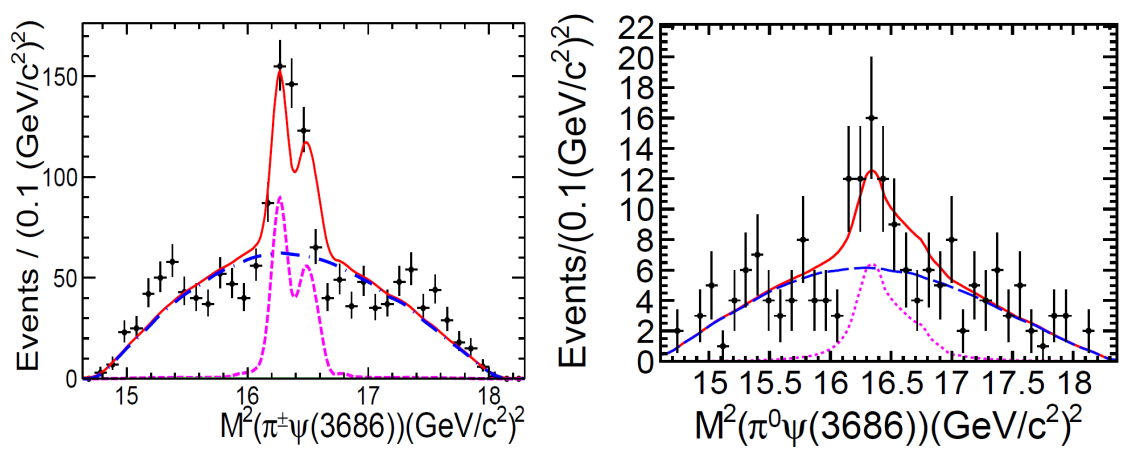

Fig. 5. Projection of $M^{2}\left(\pi^{ \pm} \psi^{\prime}\right)$ at $\sqrt{s}=4.416 \mathrm{GeV}$ with a requirement of $M^{2}\left(\pi^{+} \pi^{-}\right)>$ $0.3\left(\mathrm{GeV} / \mathrm{c}^{2}\right)^{2}$ (left) and $M^{2}\left(\pi^{0} \psi^{\prime}\right)$ at the same energy point (right).

\section{Summary}

BESIII provide new access to $X Y Z$ states by $e^{+} e^{-}$annihilation and our understanding of these exotic states has been significantly improved. Two isospin-triplets of $Z_{c}$ states are established and the spin-parity of $Z_{c}^{ \pm}(3900)$ is determined to be $1^{+}$. However, there are still many puzzled existed which are urgent needed to be understood. Even $Y(4260)$ state observed in $\pi^{+} \pi^{-} J / \psi$ no longer looks like a simple peak. The nature of these states still remains a mystery. BESIII will collect more data for $X Y Z$ study, and more exciting results of BESIII will come up soon. 


\section{Acknowledgments}

Supported in part by National Natural Science Foundation of China (NSFC) under contract Nos. 11235011, 11521505; the Ministry of Science and Technology of China under Contract No. 2015CB856701; and the CAS Center for Excellence in Particle Physics (CCEPP).

\section{References}

1. B. Aubert et al. [BaBar Collaboration], Phys. Rev. Lett. 95, 142001 (2005).

2. Q. He et al. [CLEO Collaboration], Phys. Rev. D 74, 091104 (2006).

3. C. Z. Yuan et al. [Belle Collaboration], Phys. Rev. Lett. 99, 182004 (2007).

4. B. Aubert et al. [BaBar Collaboration], Phys. Rev. Lett. 98, 212001 (2007).

5. X. L. Wang et al. [Belle Collaboration], Phys. Rev. Lett. 99, 142002 (2007).

6. M. Ablikim et al. [BESIII Collaboration], Phys. Rev. D 96, no. 3, 032004 (2017).

7. M. Ablikim et al., arXiv:1710.10740 [hep-ex].

8. M. Ablikim et al. [BESIII Collaboration], Phys. Rev. Lett. 118, no. 9, 092001 (2017).

9. M. Ablikim et al. [BESIII Collaboration], Phys. Rev. Lett. 118, no. 9, 092002 (2017).

10. M. Ablikim et al. [BESIII Collaboration], Phys. Rev. Lett. 114092003 (2015).

11. C. Patrignani et al. [Particle Data Group], Chin. Phys. C, 40, 100001 (2016).

12. M. Ablikim et al. [BESIII Collaboration], Phys. Rev. Lett. 110, 252101 (2013).

13. M. Ablikim et al. [BESIII Collaboration], Phys. Rev. Lett. 115, 112003 (2015).

14. M. Ablikim et al. [BESIII Collaboration], Phys. Rev. Lett. 112, no. 2, 022001 (2014).

15. M. Ablikim et al. [BESIII Collaboration], Phys. Rev. Lett. 115, no. 22, 222002 (2015).

16. M. Ablikim et al. [BESIII Collaboration], Phys. Rev. Lett. 111, 242001 (2013).

17. M. Ablikim et al. [BESIII Collaboration], Phys. Rev. Lett. 113, 212002 (2014).

18. M. Ablikim et al. [BESIII Collaboration], Phys. Rev. Lett. 112, 132001 (2013).

19. M. Ablikim et al. [BESIII Collaboration], Phys. Rev. Lett. 115, 182002 (2015).

20. M. Ablikim et al. [BESIII Collaboration], Phys. Rev. Lett. 119, 072001 (2017). 